\title{
Accretion of Planetary Material onto Host Stars
}

\author{
Brian Jackson and Joleen Carlberg
}

\begin{abstract}
Accretion of planetary material onto host stars may occur throughout a star's life. Especially prone to accretion, extrasolar planets in short-period orbits, while relatively rare, constitute a significant fraction of the known population, and these planets are subject to dynamical and atmospheric influences that can drive significant mass loss. Theoretical models frame expectations regarding the rates and extent of this planetary accretion. For instance, tidal interactions between planets and stars may drive complete orbital decay during the main sequence. Many planets that survive their stars' main sequence lifetime will still be engulfed when the host stars become red giant stars. There is some observational evidence supporting these predictions, such as a dearth of close-in planets around fast stellar rotators, which is consistent with tidal spin-up and planet accretion. There remains no clear chemical evidence for pollution of the atmospheres of main sequence or red giant stars by planetary materials, but a wealth of evidence points to active accretion by white dwarfs. In this article, we review the current understanding of accretion of planetary material, from the pre- to the post-main sequence and beyond. The review begins with the astrophysical framework for that process and then considers accretion during various phases of a host star's life, during which the details of accretion vary, and the observational evidence for accretion during these phases.
\end{abstract}

\section{Where are Planetary Bodies Disrupted?}

Owing to detection biases, exoplanet discoveries skew heavily toward short-period or close-in planets, as shown in Figure 1, and the nearest a planet can orbit its star

Brian Jackson

Boise State University, Dept. of Physics, 1910 University Drive, Boise, Idaho, USA 83725-1570, e-mail: bjacksoneboisestate.edu

Joleen Carlberg

Space Telescope Science Institute e-mail: jcarlberg@stsci.edu 


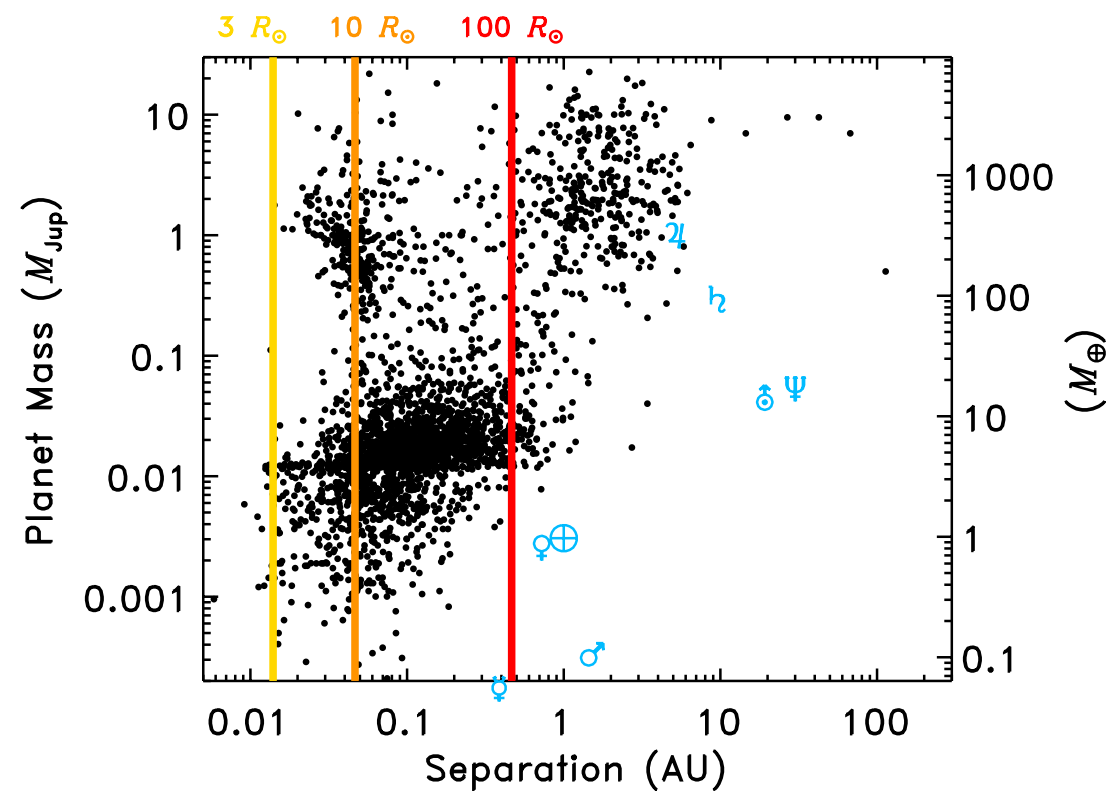

Fig. 1 Masses and separation of known exoplanets (Han et al. 2014). Blue symbols denote Solar System planets. Vertical lines show the size of the stellar radius at various stages of the post-main sequence for a Sun-like star.

and still remain intact depends largely on its self-gravity and, for smaller bodies, its tensile strength, friction, and/or internal viscosity. The Roche potential plays a key role either way, and a considerable literature has developed around the Roche potential (Kopal 1959; Paczyński|1971;: Lai et al. 1993; , Murray and Dermott|1999).

The Roche potential is the potential field around a gravitating binary system in a bound orbit (Figure 2) and is cast in a frame that rotates with the binary and so includes a centrifugal term. The potential is dominated by the planet's gravity near the planet, but moving radially outward, the planet's gravity drops off until the potential reaches a local maximum, which corresponds to zero net acceleration. The corresponding surface around the planet is defined as the Roche lobe (Murray and Dermott 1999). Such a surface also surrounds the star.

The coordinate reference frame revolves with the system and is centered on the planet with the $x$-axis pointing from the planet to the star (which are separated by a fixed orbital distance $a$ ) and the $z$-axis pointing parallel to the orbital angular momentum. Approximating the two bodies as point masses, the effective potential (gravitational + centrifugal) field $\Phi$ is

$$
\Phi=-\frac{G M_{\mathrm{p}}}{|\mathbf{r}|}-\frac{G M_{\mathrm{s}}}{|a \hat{x}-\mathbf{r}|}-\frac{1}{2} \Omega^{2}\left[\left(x-x_{\mathrm{cm}}\right)^{2}+y^{2}\right],
$$




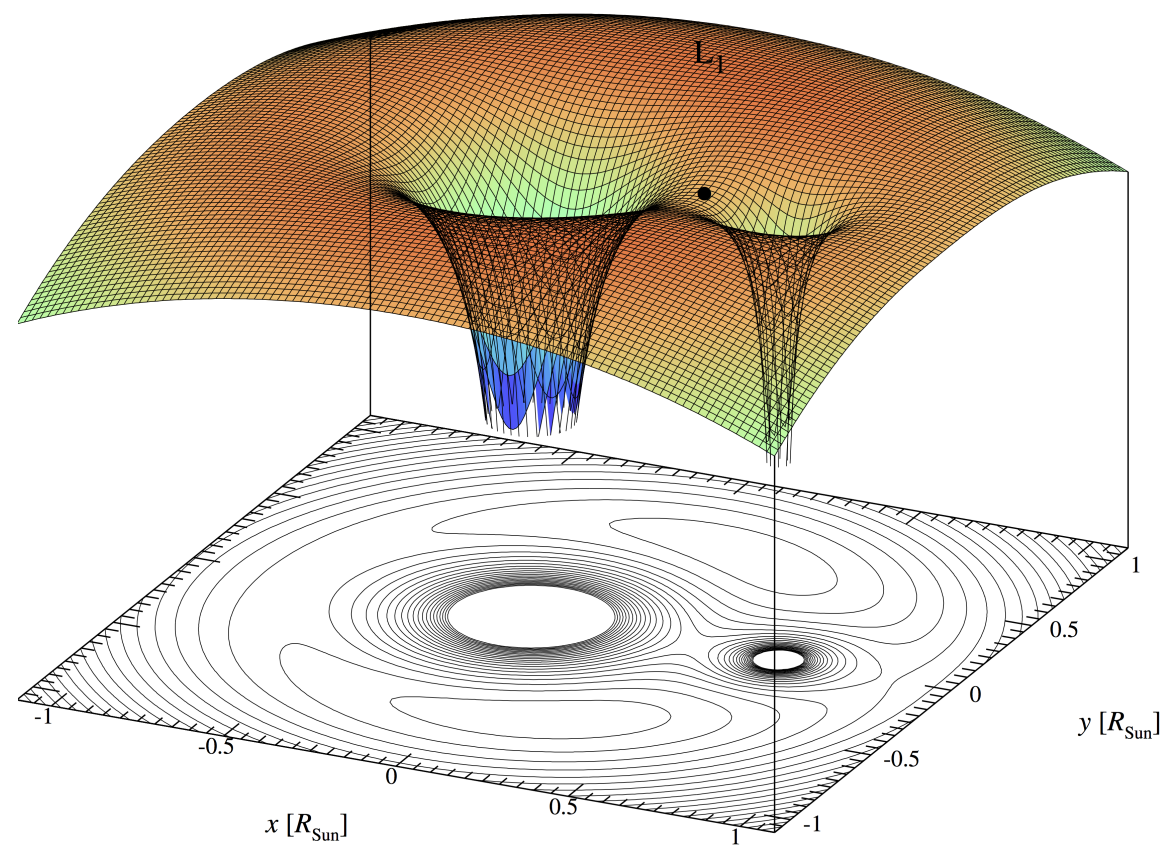

Fig. 2 The Roche potential. The colored grid shows a three-dimensional representation of the potential surface in the $x-y$ plane. Contours at the bottom show a projection of isopotentials onto the $x-y$ plane. The Lagrange point L1 is labeled. For this figure, $q=0.2$ and distances are measured in solar radii $R_{\text {Sun }}$, with the two masses separated by $0.718 R_{\text {Sun }}$. Courtesy of René Heller.

where $G$ is the gravitational constant, $M_{\mathrm{s}} / M_{\mathrm{p}}$ is the stellar/planetary mass, $\mathbf{r}$ the location at which to evaluate $\Phi$, and $\hat{x}$ a unit vector pointing along $x$. The system's center of mass is at $x_{\mathrm{cm}}=a\left[M_{\mathrm{s}} /\left(M_{\mathrm{s}}+M_{\mathrm{p}}\right)\right] . \Omega$ is the orbital mean motion, and $\Omega^{2}=G\left(M_{\mathrm{p}}+M_{\mathrm{s}}\right) / a^{3}$.

The Roche lobe is not spherical, and there is no general closed form expression for that surface. However, Eggleton (1983) provided a formula for the volumeequivalent radius of the Roche lobe $R_{\mathrm{RL}}$, i.e., the radius of a sphere with a volume equivalent to the given Roche lobe's, accurate to $1 \%$ for all mass ratios $q=M_{\mathrm{p}} / M_{\mathrm{s}}$ :

$$
R_{\mathrm{RL}}=\frac{0.49 q^{2 / 3}}{0.6 q^{2 / 3}+\ln \left(1+q^{1 / 3}\right)} a
$$

and for $q \ll 1$

$$
R_{\mathrm{RL}} \approx 0.49 q^{1 / 3} a .
$$

For planets (and stars) composed of slowly moving fluid, contours of $\Phi$ coincide closely with density contours, and so the surface of a planet just filling its Roche lobe will correspond very nearly to the Roche lobe. To decide whether a planet is losing mass, we can compare the planet's mean radius $R_{\mathrm{p}}$ to $R_{\mathrm{RL}}$. Incorporating Ke- 
pler's Third Law, Rappaport et al. (2013) recast Equation 3 to estimate the orbital period for the Roche limit, $P_{\mathrm{RL}}$, from the corresponding semi-major axis, $a_{\mathrm{RL}}$, for a planet with a bulk density $\rho_{\mathrm{p}}$ :

$$
P_{\mathrm{RL}} \approx \sqrt{\frac{3 \pi}{(0.49)^{3} G \rho_{\mathrm{p}}}} \approx 9.6 \mathrm{hrs}\left(\frac{\rho_{\mathrm{p}}}{1 \mathrm{~g} \mathrm{~cm}^{-3}}\right)^{-1 / 2} .
$$

Figure 3 compares the current periods $P$ to $P_{\mathrm{RL}}$ for several short-period planets.

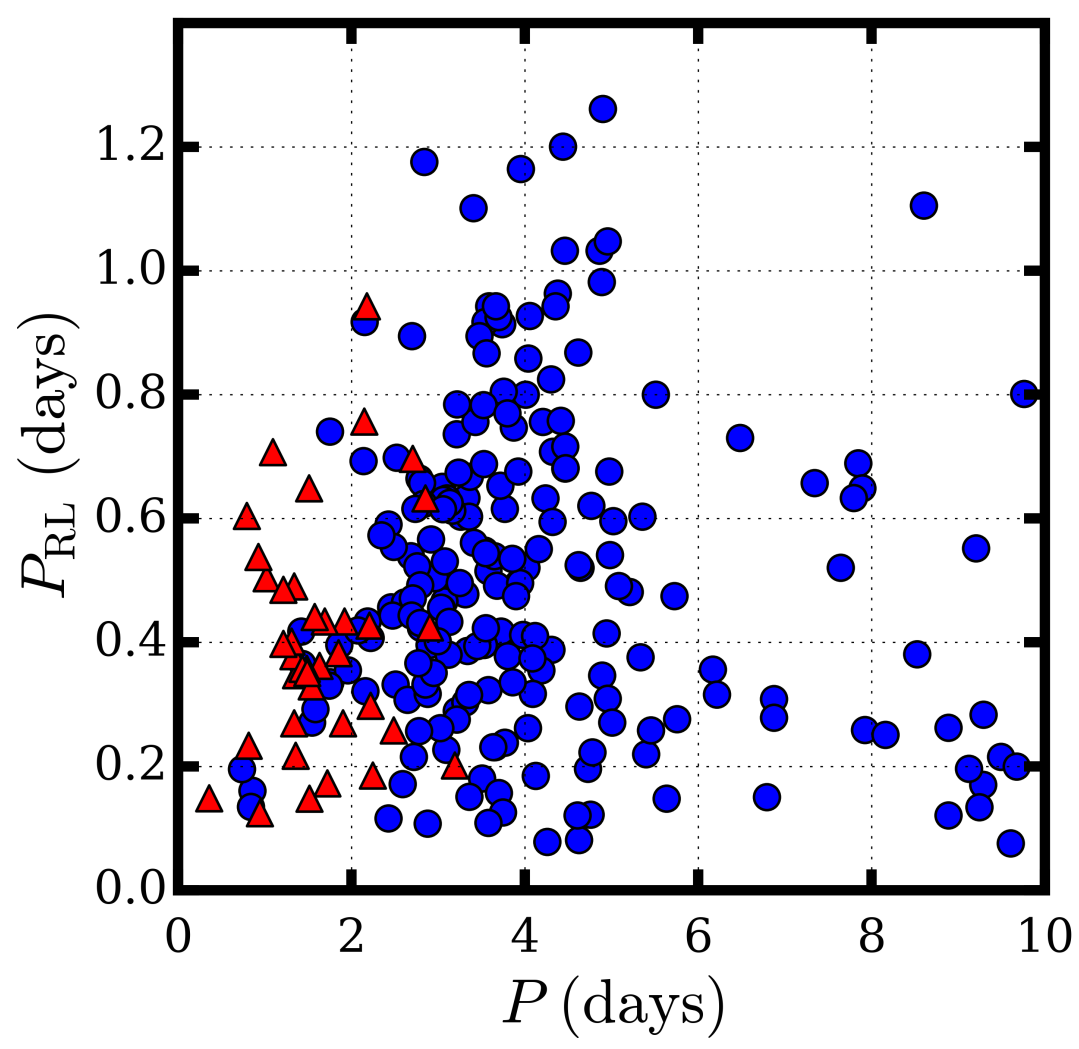

Fig. 3 Roche limit periods $P_{\text {RL }}$ (Equation 4 vs. orbital periods $P$ for many short-period planets. Red triangles indicate planets for which tidal interactions with the host star may bring a planet with zero orbital eccentricity into its Roche limit in less than $1 \mathrm{Gyr}$ (assuming a modified tidal dissipation parameter for the star $Q_{\mathrm{s}}^{\prime}=10^{7}$-Penev et al. 2012 - see "Tides in star-planet systems" chapter.

Importantly, the classical Roche limit here involves a number of assumptions, including treating the planet and star as point masses, assuming a circular orbit (Jackson et al. 2008) and synchronized planetary rotation (Showman and Guillot 2002), 
neglecting the effects of additional bodies (Van Laerhoven and Greenberg |2012, Hansen and Zink 2015), and neglecting tensile strength and friction (Richardson et al. 1998; Davidsson 1999).

\section{How are Planetary Bodies Disrupted?}

Once some portion of the planet's surface or atmosphere contacts the Roche lobe, it will begin losing mass (Roche-lobe overflow or RLO). Whether RLO proceeds stably or not depends on how the planet and its orbit respond to the mass loss. For dense planets with $a_{\mathrm{RL}}$ below the stellar photosphere, other processes determine how the planet is disrupted.

\section{Roche-Lobe Overflow}

For short-period exoplanets, tidal interactions with the host star usually transfer orbital angular momentum to the star's rotation and reduce the semi-major axis. Even for zero eccentricity, tides can bring hot Jupiters $(P \sim$ days $)$ into Roche-lobe contact within Gyrs (Jackson et al. 2008). Smaller planets raise smaller tides, driving slower orbital decay, but Earth-sized, rocky planets have been found with $P \sim$ hrs and may also be accreted in Gyrs (Sanchis-Ojeda et al.|2013; Jackson et al. 2013, Sanchis-Ojeda et al. 2014, Adams et al. 2016).

Material leaks out primarily through the L1 Lagrange point between the planet and the star. As the mass escapes, conservation of angular momentum can form a thin accretion disk around the star. Viscous stresses within the disk and tidal interactions with the disrupting planet can transfer some of the disk's angular momentum back to the planet, driving the material inward (Ritter 1988).

Torques between the planet and disk act in the opposite direction as the torque from the stellar tide (Lin and Papaloizou 1979), but mass loss would stop if the planet stopped filling its Roche lobe. Consequently, the disk torque cannot drive the planet beyond the Roche limit. However, if the tide raised on the host star pulled the planet inward through the Roche limit, the mass loss would increase (Ritter 1988), increasing the mass in the accretion disk and the strength of the disk's torque. The resulting balance, stable RLO (Priedhorsky and Verbunt 1988), keeps the planet's radius $R_{\mathrm{p}} \approx R_{\mathrm{RL}}$. If the planet's density drops as it loses mass, $P_{\mathrm{RL}}$ increases (Equation 4), and the gas disk would drive the planet out with the Roche limit (Valsecchi et al. 2015; Jackson et al. 2016).

This balance relates the planet's orbital evolution to its mass and radius evolution:

$$
\left(\frac{\dot{M}_{\mathrm{p}}}{M_{\mathrm{p}}}\right)=-\frac{1}{2} \eta^{-1}\left[\frac{1}{a}\left(\frac{\partial a}{\partial t}\right)_{\mathrm{star}}+\left(\frac{\partial \ln R_{\mathrm{p}}}{\partial t}\right)_{\mathrm{M}_{\mathrm{p}}}\right],
$$




$$
\left(\frac{\dot{a}}{a}\right)=\eta^{-1}\left[\left(\frac{1}{3}-\xi\right) \frac{1}{a}\left(\frac{\partial a}{\partial t}\right)_{\mathrm{star}}+(1-\delta \gamma)\left(\frac{\partial \ln R_{\mathrm{p}}}{\partial t}\right)_{\mathrm{M}_{\mathrm{p}}}\right]
$$

where $M_{\mathrm{p}} \ll M_{\mathrm{s}}$.

Here, $\xi=\partial \ln R_{\mathrm{p}} / \partial \ln M_{\mathrm{p}}$. The escaping gas has a specific angular momentum $\gamma \sqrt{G M_{\mathrm{s}} a}$, but the angular momentum is not necessarily returned to the planet. Gas may escape from the system, driven by stellar wind (Bloecker 1995), or angular momentum is transferred to the star during accretion. The parameter $\delta$ expresses the fraction of gas that does not return its angular momentum: $\delta=0$ means all is returned, while $\delta=1$ means none is. These parameters combine to give $\eta=$ $\xi / 2-\delta \gamma+5 / 6$ (5/6 comes from the Roche lobe's mass dependence).

A vast literature exists that provides many formulations for tidal interactions in binary systems (see Ferraz-Mello et al. 2008 for a thorough review). One well-worn model (Goldreich and Soter 1966) gives

$$
\left(\frac{\partial a}{\partial t}\right)_{\mathrm{star}}=-\frac{9}{2}\left(\frac{G}{M_{\mathrm{s}}}\right)^{1 / 2} \frac{R_{\mathrm{s}}^{5} M_{\mathrm{p}}}{Q_{\mathrm{s}}^{\prime}} a^{-11 / 2}
$$

where $Q_{\mathrm{s}}^{\prime}$ is the modified tidal dissipation parameter (Ferraz-Mello et al.|2008). Unfortunately, the details of tidal dissipation for stars and gaseous planets are not well understood, and predictions for $Q_{\mathrm{s}}^{\prime}$ span a wide range (cf. Ogilvie 2014). Consequently, the timescales for tidal decay and RLO are poorly known, but the outcomes for stable RLO should be insensitive to the rates.

With a mass-radius relation, Equations 5 and 6 can be integrated to model the evolution of a disrupting gaseous planet. Valsecchi et al. (2015) and Jackson et al. (2016) employed the Modules in Stellar Astrophysics (MESA) suite (Paxton et al. 2011, 2013, 2015) to model disrupting hot Jupiters with a variety of initial conditions, and Figure 4 illustrates the results. For a hot Jupiter $\xi \approx 0$ (Fortney et al. 2007), so mass loss initially reduces $\rho_{\mathrm{p}}$, increases $P_{\mathrm{RL}}$, and drives planets out. Eventually, mass loss removes most of the gaseous envelope, $\rho_{\mathrm{p}}$ and $P_{\mathrm{RL}}$ drop, and the tide can draw the planet back in. The outcome depends on the mass of the planet's solid core, not on initial conditions (assuming they allow for RLO): the smaller the core, the more orbital expansion.

Fig. 4 Figure 4 from Jackson et al. (2016). Not included due to copyright restrictions.

Jackson et al. (2016) found that the gaseous planets that reach their Roche limits can completely shed their atmospheres, resulting in significant orbital expansion (which slows disruption and may save the remnant from accretion) or rapid accretion of the planet by the star.

Stable RLO requires a specific relationship between evolution of $R_{\mathrm{p}}$ and of $R_{\mathrm{RL}}$ :

$$
\delta \gamma<\xi / 2+5 / 6
$$


If this inequality is not satisfied, unstable RLO may result. For example, if $\gamma \approx 1$ and $\xi \approx 0$, stability requires no more than $5 / 6(=0.8 \overline{3})$ of the mass escaping from the planet be lost without returning its angular momentum. Loss of orbital angular momentum is inevitable - Metzger et al. (2012) pointed out that material accreted at the stellar surface carries non-negligible angular momentum which will not be returned to the orbit, translating into $\delta \gamma=\sqrt{R_{\star} / a_{\mathrm{RL}}}$. For Jupiter orbiting the Sun, $a=a_{\text {Roche }} \approx 0.01 \mathrm{AU} \approx 2 R_{\text {Sun }}$, and $\delta \gamma=\sqrt{1 / 2}$.

Valsecchi et al. (2015) explored loss of escaped material and found instability was likely, while Jia and Spruit (2017) found rocky planets between 1 and 10 Earth masses $M_{\text {Earth }}$ are likely to undergo unstable mass transfer. During unstable RLO, the mass loss timescale is comparable to the orbital period, but what happens next is unclear. Valsecchi et al. (2015) speculated that the escaped material may form a torus orbiting the host star, and interactions with the denuded planet may eject the torus, driving the planet into its Roche limit or the star.

\section{Disruption Below the Stellar Photosphere}

The photosphere for a Sun-like, main sequence star corresponds to the Roche limit for $\rho_{\mathrm{p}}=8 \mathrm{~g} \mathrm{~cm}^{-3}$, denser than nearly all super-Earths found by the Kepler mission (Weiss and Marcy 2014). Planets orbiting lower density, red giant stars, though, may enter the stellar photosphere relatively unscathed - Figure 1.

Several outcomes are possible for such planets. The most massive companions may unbind the stellar envelopes, but only for asymptotic giant branch stars, when stars reach their largest radii and thus lowest binding energy (Nordhaus and Blackman 2006). In smaller red giants, companions can (1) enter into a common envelope stage if $M_{\mathrm{p}} \geq 5-20 M_{\mathrm{Jup}}$, with the final mass of the accreting companion dependent on the star's envelope mass; (2) fully evaporate in the stellar envelope; or (3) impact the stellar core (Livio and Soker 1984) .

For the latter two cases, evaporation within the star will occur near the depth where the temperature of the stellar interior exceeds the virial temperature of the planet $T_{\mathrm{v}}$ (Siess and Livio|1999a):

$$
T_{\mathrm{v}}=\frac{G M_{\mathrm{p}} \mu_{\mathrm{p}} m_{\mathrm{H}}}{k R_{\mathrm{p}}}
$$

where $\mu_{\mathrm{p}}$ is the planet's mean molecular weight, $m_{\mathrm{H}}$ hydrogen's mass, and $k$ Boltzmann's constant. Aguilera-Gómez et al. (2016) modeled dissolution for a range of companion planets and brown dwarfs and stellar masses and metallicities, and found that companions with $M_{\mathrm{p}} \leq 15 M_{\text {Jup }}$ will evaporate in the stellar envelope but more massive companions would probably impact the stellar core. 


\section{When are Planetary Bodies Disrupted?}

Planetary accretion may occur from the pre-main sequence through the white dwarf stage, and different physical processes drive the accretion in different stages. The composition and rotation of a host star and orbital architecture of a planetary system may be long-lived signatures of planetary accretion, although unequivocal evidence is lacking for many stages.

\section{During the Pre- and Main Sequence}

In principle, accretion of planetary material changes a star's composition, an idea originally invoked to explain the planet-metallicity correlation (Fischer and Valenti 2005). The stellar mass fraction $X_{i}$ for an element $i$ is given as Siess and Livio (1999b)

$$
X_{i}=\frac{X_{i}^{\mathrm{CZ}} M_{\mathrm{CZ}}+X_{i}^{\mathrm{acc}} M_{\mathrm{acc}}}{M_{\mathrm{acc}}+M_{\mathrm{CZ}}},
$$

where ' $\mathrm{CZ}$ ' refers to the stellar convection zone and 'acc' to the accreted material, and $M$ is the associated total mass.

The changes to the photospheric abundances depend on the amount of accreted material and how much its composition differs from the star's. For hydrogendominated, giant planets, the least stellar-like compositions are found in their cores. The convection zone in Sun-like stars makes up a small fraction of the stars' masses, and so a little material can cause large local abundance enhancements. The fact that the planet-metallicity correlation does not depend on convective envelope mass strongly suggests the correlation does not arise from planetary accretion (Fischer and Valenti 2005).

However, accretion during the pre- and main sequence may impart other chemical signatures. Recent work suggests the last gas accreted onto stars is depleted in planet-forming materials, which are probably locked-up in terrestrial planets or gas giant's cores. Meléndez et al. (2009) and Ramírez et al. (2009) found that the absence of $\sim 4 M_{\oplus}$ of rocky material could explain a depletion in refractory elements in the Sun's photosphere compared to solar twins, fueling research into abundance comparisons between binary stars for which only one hosts a planet. For example, the 16 Cyg binary system is composed of two solar analogs, and one hosts a planet (Cochran et al. 1997). The co-evality and nearly identical masses of the stars makes 16 Cyg ideal for measuring abundance differences, and Tucci Maia et al. (2014) found a telling deficiency in the planet-hosting star corresponding to $1.5-6 M_{\oplus}$ of missing metals.

Planetary accretion may induce downward mixing (Théado and Vauclair 2012), effectively erasing expected abundance enhancements (Equation 10) but propitiously, this mixing can also create a new signature. The convection zone is the only region in Sun-like stars where fragile elements, like lithium, can survive, and 
downward mixing carries those elements to hotter regions, where they are destroyed, permanently depleting their surface abundances. Deal et al. (2015) argued that this scenario could explain the discrepant lithium abundances in the 16 Cyg system if the planet-hosting star, which has less lithium, accreted less than an Earth's mass of material.

Some migration likely brings short-period planets in from where they form, and two types have been considered: gas disk migration and dynamical excitation, followed by tidal circularization. In later stages, planets may enjoy long-lived stability, but, for short-period planets, tidal interactions can drive significant orbital decay Figure 3 . Additional evidence for planetary accretion may come from considering these dynamical processes.

\section{Due to Gas Disk Migration}

Gas disk migration involves gravitational interactions between a planet and its disk and can drive significant orbital evolution during the disk's lifetime, $\leq 10$ Myrs (Bell et al. 2013: Trilling et al. 1998; Chambers 2009). Lin et al. (1996) suggested a young star's magnetosphere might clear the protoplanetary disk several stellar radii from the star, allowing gas disk migration to deposit planets in orbits near the disk's inner edge (Lin and Papaloizou 1979, Goldreich and Tremaine 1979). Coupling between the magnetosphere and disk may synchronize the star's rotation to the innermost disk (Koenigl 1991); thus, a young star's rotation period may indicate the orbital period of the disk's inner edge and the stopping point for gas disk migration. Irwin and Bouvier (2009) found young stars' rotation periods range from $<1$ day up to $\sim 10$ days, suggesting that disk migration may bring planets very close in. Indeed, van Eyken et al. (2012) discovered a possible hot Jupiter in a 10-hour orbit around a T Tauri star, likely in RLO (Jackson et al.2017).

\section{Due to Dynamical Excitation}

Under an alternative scenario, short-period planets formed in roughly circular orbits with initial semi-major axes $\geq 1 \mathrm{AU}$. Interactions with other planets or companion stars via Kozai resonances (Zuckerman et al. 2007; Naoz 2016) or dynamical instabilities (Rasio and Ford 1996; Chatterjee et al. 2008) excited the planets' orbital inclinations and eccentricities to large values, giving small enough pericenters for tidal circularization to shrink the orbits. Given the potentially chaotic nature of these interactions, production of short-period planets is only one outcome (Matsumura et al. 2008; Nagasawa et al. 2008). Often, a planet is scattered into a Roche-limit crossing orbit, resulting in disruption and accretion (Guillochon et al. 2011; Liu et al. 2013). Since tidal circularization is dominated by dissipation within the planet (Jackson et al.2008), it can also heat and inflate a gaseous planet to its Roche limit (Gu et al.2003). 
Since dynamical excitation can significantly misalign the stellar rotation and orbital angular momentum vectors, the spin-orbit alignment for planetary systems may reveal their histories. For many systems, Albrecht et al. (2012) compiled prior and new estimates of stellar obliquity angles as projected on the plane of the sky (cf. Fabrycky and Winn 2009) and found the angles were more broadly distributed for hotter stars than for cooler stars. Since tidal interactions may be more effective at reducing obliquities for cooler than hotter stars, this distribution may point to the dynamical excitation mode of short-period planet production (Winn et al. 2010). Other interpretations of the obliquity distribution do not require the dynamical excitation (and concomitant accretion) (Batygin 2012, Huber et al. 2013).

Other aspects of the orbital architectures of planetary systems may also suggest planetary accretion via dynamical excitation. Mandell et al. (2007) demonstrated that material around a soon-to-be hot Jupiter migrating through a gas disk can coalesce into low-mass companions, but Mustill et al. (2015) pointed out that dynamical excitation of the planet would instead empty the inner system of low-mass planets by driving many into the host star. The apparent lack of sibling planets in hot Jupiter systems supports this latter scenario (Steffen et al. 2012), although the idea that hot Jupiters tend to be "lonely" has been challenged (Schlaufman and Winn 2016).

\section{Due to Tidal Decay}

After the disk has dissipated, tidal interactions with a short-period planet and loss of angular momentum via stellar wind dominate a star's rotational evolution: the former tends to synchronize the rotation with the planet's orbital motion, usually increasing the spin rate (Mardling and Lin 2002, Jackson et al. 2008, Brown et al. 2011), while the latter slows the spin (Skumanich 1972; Stauffer and Hartmann 1987: Kawaler 1988, Barnes and Sofia 1996). Drawing on Counselman (1973), Levrard et al. (2009) and Adams and Bloch (2015) showed the total angular momentum (rotational + orbital) for many exoplanetary systems lies below a threshold value for stability, meaning those planets are doomed to RLO or accretion - Figure 5 Since this result holds whatever the rate of tidal decay, determining whether decay occurs on Gyr timescales requires additional observations.

Fig. 5 Figure 3 from Adams and Bloch 2015. Not included due to copyright restrictions.

Ford and Rasio (2006) pointed out that the dynamical excitation production mode should give an initial cut-off in orbital semi-major axes at twice the Roche limit, and the distribution does show such a cut-off, albeit with some of the shortestperiod planets interior to this cut-off. Valsecchi and Rasio (2014) interpreted this distribution as evidence for tidal decay subsequent to dynamical excitation and tidal circularization.

Evidence of tidal spin-up and planetary engulfment also appears in the distribution of planetary orbits for stars of different rotation periods. McQuillan et al. 
(2013) measured rotation periods of main sequence planet hosts observed by the Kepler mission and found that the faster the stars rotated, the farther out the innermost planets were found. Teitler and Königl (2014) interpreted this relationship as evidence that the close-in planets of those fast-rotating stars had been engulfed.

\section{During the Post-Main Sequence}

During the red giant phase, expansion of the stellar envelope enhances tidal decay of planetary orbits (Equation 7), which competes with orbital expansion from stellar mass loss. Figure 1 shows that many known exoplanets may eventually be engulfed, and numerous studies have explored the survival limits around red giants (e.g., Villaver and Livio 2007, 2009; Carlberg et al. 2009; Kunitomo et al.2011). A corollary of this observation is that many current red giant stars have engulfed one or more planetary companions.

Siess and Livio (1999b) was one of the earliest comprehensive calculations of the signatures of planet engulfment by an evolved solar-type star. They modeled engulfment as an accretion rate deep in the star and predicted enhanced rotation rates, changes to the composition of the stellar atmosphere, reemergence of a dynamo with associated X-ray emission, and ejection of shellular material with associated infrared emission.

The rotation rates of red giants are generally slow and unmeasurable in many cases. Enhanced rotation is thus a telltale signature of engulfment, provided that the stellar mass is known to be low (since low-mass stars are slow main sequence rotators) and that interactions with stellar companions can be ruled out. Carlberg et al. (2009) demonstrated that known exoplanet systems have sufficiently large angular momentum that planetary accretion could create rapid stellar rotation on the red giant branch. However, this fast rotation signature will not persist throughout the red giant phase because of the steadily increasing stellar radius. Furthermore, its detection requires a favorable orientation of the stellar rotation axis to the observer's line of sight.

Surface composition changes are expected to be modest for engulfment by evolved stars because of their large convection zones (large $M_{\mathrm{CZ}}$ in Equation 10]. A notable exception is the group of fragile elements, $\mathrm{Li}, \mathrm{Be}$, and $\mathrm{B}$, which are destroyed by proton-capture reactions throughout the star's life and should be depleted in the atmospheres of red giants that have not engulfed a planet. Lithium is the element most affected by this process. For stars undergoing simple first-dredge up mixing, the depletion factor is approximately a few tens but can rise to 100,000 for deep circulation between the stellar envelope and the interior (Sackmann and Boothroyd 1999). In such stars, the amount of Li deposited in the stellar envelope by an engulfed planet could greatly exceed the Li already present.

Alexander (1967) first predicted that engulfed planets could increase the surface $\mathrm{Li}$ in red giants, and Li-enriched red giants were subsequently discovered (Wallerstein and Sneden 1982). However, some of these red giants have Li exceeding ex- 
pectations from engulfment. Paradoxically, stars with deep circulation may be able to replenish the surface lithium. Stars synthesize $\mathrm{Li}$ from ${ }^{3} \mathrm{He}$ at depths where the $\mathrm{Li}$ is almost instantly destroyed, but fast circulation of material between these depths and the surface can conceivably salvage newly created $\mathrm{Li}$ (Sackmann and Boothroyd 1999). Thus, the ability to use $\mathrm{Li}$ as an engulfment signature is limited by how well a given red giant star's expected $\mathrm{Li}$ abundance can be constrained.

Although identifying individual planet-engulfing red giants remains elusive, evidence of engulfment appears in ensemble studies. Drake et al. (2002) noted that fast-rotating red giants were much more likely to be Li-rich than slow red giants, and Carlberg et al. (2012) demonstrated a global enhancement of $\mathrm{Li}$ in fast-rotators, consistent with simultaneous increases in both rotation and Li from engulfment.

Further evidence for engulfment may be seen in the distribution of planetary companions to evolved stars: there is a deficiency in short-period companions (with semi-major axes between 0.06 to $0.5 \mathrm{AU}$ ) compared to main sequence populations. However, the extent of the deficiency may not be consistent with tidal engulfment since many red giants have not grown large enough to engulf planets out to $0.5 \mathrm{AU}$. A few recent discoveries, notably HIP13044 at 0.116 AU (Setiawan et al. 2010) and Kepler-91 at 0.07 AU (Lillo-Box et al. 2014), include planets passing within a few stellar radii at pericenter passage. In any case, the protracted desert of planets between 0.06 and $0.5 \mathrm{AU}$ around evolved stars remains unexplained.

\section{In the Stellar Graveyard}

Not even stellar death can stop the accretion of planetary material. Roughly the size of Earth but with masses comparable to the Sun, white dwarfs are extremely dense $\left(\geq 10^{4} \mathrm{~g} / \mathrm{cm}^{3}\right)$ and hot $(\sim 10,000 \mathrm{~K})$ objects primarily composed of carbon and oxygen covered with thin, stratified atmospheres of helium and hydrogen. Given the large densities of white dwarfs, disruption of planetary bodies likely results in the formation of an accretion disk, with the disk materials transitioning from solid particles in the outermost regions to vapor in the innermost regions nearest the white dwarf (Veras et al. 2014).

The observational evidence supporting accretion of planetary material onto white dwarfs is more robust than for any other stage of stellar evolution. Elements heavier than He should settle out of the atmosphere in just weeks or less due to the white dwarf's strong gravitational field, except in white dwarfs hotter than $\sim 20,000 \mathrm{~K}$ (Chayer et al. 1995), where radiative levitation counteracts the settling. Nevertheless, absorption features from metals have been detected in white dwarfs cooler than $20,000 \mathrm{~K}$, and these metals must have been recently or are continually being accreted. The sources of these metals are thought to be disrupted asteroids and/or comets (Zuckerman et al. 2007), an idea supported by the infrared excesses seen around white dwarfs. The outer radii of the disks inferred from the infrared are $\sim R_{\odot}$, or approximately the Roche limit for asteroidal bodies (Jura 2003). How these asteroids and comets are injected into Roche-lobe crossing orbits remains un- 
clear, but the shifting of resonant interactions between a distant asteroid or Kuiper belt and a perturbing planet as the result of stellar mass loss may be responsible (Bonsor et al.|2011; Debes et al.|2012).

The measured relative abundances provide estimates of the bulk composition of the accreted material, and the resulting elemental ratios can be compared to classes of objects in the solar system to infer the properties of the accreted object. For example, relative surpluses or deficiencies in Fe to Si have been interpreted as accreted cores or crusts of a differentiated body. There is even evidence for the accretion of water-rich planetary debris, discovered by accounting for the various mineralogical carriers of oxygen (e.g., $\mathrm{SiO}_{2}$ ) and attributing the observed excess $\mathrm{O}$ to $\mathrm{H}_{2} \mathrm{O}$ (Farihi et al.2013).

Acknowledgements This work has made use of the Exoplanet Orbit Database and the Exoplanet Data Explorer at exoplanets.org.

\section{References}

Adams ER, Jackson B Endl M (2016) Ultra-short-period Planets in K2 SuPerPiG Results for Campaigns 0-5. AJ152:47

Adams FC Bloch AM (2015) On the stability of extrasolar planetary systems and other closely orbiting pairs. Monthly Notices of the Royal Astronomical Society 446:3676-3686, URL http://dx.doi.org/10.1093/mnras/stu2397

Aguilera-Gómez C, Chanamé J, Pinsonneault MH Carlberg JK (2016) On Lithium-rich Red Giants. I. Engulfment of Substellar Companions. The Astrophysical Journal 829(2):127

Albrecht S, Winn JN, Johnson JA et al. (2012) Obliquities of Hot Jupiter Host Stars: Evidence for Tidal Interactions and Primordial Misalignments. ApJ757:18

Alexander JB (1967) A possible source of lithium in the atmospheres of some red giants. The Observatory 87:238-240

Barnes S Sofia S (1996) On the Origin of the Ultrafast Rotators in Young Star Clusters. ApJ462:746

Batygin K (2012) A primordial origin for misalignments between stellar spin axes and planetary orbits. Nature 491:418-420

Bell CPM, Naylor T, Mayne NJ, Jeffries RD Littlefair SP (2013) Pre-main-sequence isochrones II. Revising star and planet formation time-scales. Monthly Notices of the Royal Astronomical Society 434:806-831, URL http://dx.doi.org/10.1093/mnras/stt1075

Bloecker T (1995) Stellar evolution of low and intermediate-mass stars. I. Mass loss on the AGB and its consequences for stellar evolution. A\&A297:727

Bonsor A, Mustill AJ Wyatt MC (2011) Dynamical effects of stellar mass-loss on a Kuiper-like belt. MNRAS414:930-939

Brown DJA, Collier Cameron A, Hall C, Hebb L Smalley B (2011) Are falling planets spinning up their host stars? MNRAS415:605-618

Carlberg JK, Majewski SR Arras P (2009) The Role of Planet Accretion in Creating the Next Generation of Red Giant Rapid Rotators. The Astrophysical Journal 700(1):832-843

Carlberg JK, Cunha K, Smith VV Majewski SR (2012) Observable Signatures of Planet Accretion in Red Giant Stars. I. Rapid Rotation and Light Element Replenishment. The Astrophysical Journal 757(2):109

Chambers JE (2009) Planetary Migration: What Does It Mean for Planet Formation? Annual Review of Earth and Planetary Sciences 37:321-344 
Chatterjee S, Ford EB, Matsumura S Rasio FA (2008) Dynamical Outcomes of Planet-Planet Scattering. ApJ686:580-602

Chayer P, Fontaine G Wesemael F (1995) Radiative Levitation in Hot White Dwarfs: Equilibrium Theory. ApJS99:189

Cochran WD, Hatzes AP, Butler RP Marcy GW (1997) The Discovery of a Planetary Companion to 16 Cygni B. ApJ483:457-463

Counselman CC III (1973) Outcomes of Tidal Evolution. ApJ180:307-316

Davidsson BJR (1999) Tidal Splitting and Rotational Breakup of Solid Spheres. Icarus142:525535

Deal M, Richard O Vauclair S (2015) Accretion of planetary matter and the lithium problem in the 16 Cygni stellar system. A\&A 584:A105

Debes JH, Walsh KJ Stark C (2012) The Link between Planetary Systems, Dusty White Dwarfs, and Metal-polluted White Dwarfs. ApJ747:148

Drake NA, de la Reza R, da Silva L Lambert DL (2002) Rapidly Rotating Lithium-rich K Giants: The New Case of the Giant PDS 365. The Astronomical Journal 123(5):2703-2714

Eggleton PP (1983) Approximations to the radii of Roche lobes. ApJ268:368

Fabrycky DC Winn JN (2009) Exoplanetary Spin-Orbit Alignment: Results from the Ensemble of Rossiter-McLaughlin Observations. ApJ696:1230-1240

Farihi J, Gänsicke BT Koester D (2013) Evidence for Water in the Rocky Debris of a Disrupted Extrasolar Minor Planet. Science 342:218-220

Ferraz-Mello S, Rodríguez A Hussmann H (2008) Tidal friction in close-in satellites and exoplanets: The Darwin theory re-visited. Celestial Mechanics and Dynamical Astronomy 101:171201

Fischer DA Valenti J (2005) The Planet-Metallicity Correlation. ApJ622:1102-1117

Ford EB Rasio FA (2006) On the Relation between Hot Jupiters and the Roche Limit. ApJ638:L45L48

Fortney JJ, Marley MS Barnes JW (2007) Planetary radii across five orders of magnitude in mass and stellar insolation: Application to transits. Astrophys J 659(2):1661-1672, URL http: //dx.doi.org/10.1086/512120

Goldreich P Soter S (1966) Q in the Solar System. Icarus5:375-389

Goldreich P Tremaine S (1979) The excitation of density waves at the Lindblad and corotation resonances by an external potential. ApJ233:857-871

Gu PG, Lin DNC Bodenheimer PH (2003) The Effect of Tidal Inflation Instability on the Mass and Dynamical Evolution of Extrasolar Planets with Ultrashort Periods. ApJ588:509-534

Guillochon J, Ramirez-Ruiz E Lin D (2011) Consequences of the Ejection and Disruption of Giant Planets. ApJ732:74

Han E, Wang SX, Wright JT et al. (2014) Exoplanet Orbit Database. II. Updates to Exoplanets.org. PASP126:827

Hansen BMS Zink J (2015) On the potentially dramatic history of the super-Earth $\rho 55$ Cancri e. MNRAS450:4505-4520

Huber D, Carter JA, Barbieri M et al. (2013) Stellar Spin-Orbit Misalignment in a Multiplanet System. Science 342:331-334

Irwin J Bouvier J (2009) The rotational evolution of low-mass stars. In: Mamajek EE, Soderblom DR Wyse RFG (eds) The Ages of Stars, IAU Symposium, vol 258, pp 363-374, DOI 10.1017/ S1743921309032025

Jackson B, Greenberg R Barnes R (2008) Tidal Evolution of Close-in Extrasolar Planets. ApJ678:1396-1406

Jackson B, Stark CC, Adams ER, Chambers J Deming D (2013) A Survey for Very Short-period Planets in the Kepler Data. ApJ779:165

Jackson B, Jensen E, Peacock S, Arras P Penev K (2016) Tidal decay and stable Roche-lobe overflow of short-period gaseous exoplanets. Celestial Mechanics and Dynamical Astronomy $126: 227-248$

Jackson B, Arras P, Penev K, Peacock S Marchant P (2017) A New Model of Roche Lobe Overflow for Short-period Gaseous Planets and Binary Stars. ApJ835:145 
Jia S Spruit HC (2017) Instability of mass transfer in a planet-star system. MNRAS465:149-160

Jura M (2003) A Tidally Disrupted Asteroid around the White Dwarf G29-38. The Astrophysical Journal 584(2):L91-L94

Kawaler SD (1988) Angular momentum loss in low-mass stars. ApJ333:236-247

Koenigl A (1991) Disk accretion onto magnetic T Tauri stars. ApJ370:L39-L43

Kopal Z (1959) Close binary systems

Kunitomo M, Ikoma M, Sato B, Katsuta Y Ida S (2011) Planet Engulfment by $1.5-3 \mathrm{M}_{\text {sun }}$ Red Giants. ApJ737:66

Lai D, Rasio FA Shapiro SL (1993) Ellipsoidal figures of equilibrium - Compressible models. ApJS88:205-252

Levrard B, Winisdoerffer C Chabrier G (2009) Falling Transiting Extrasolar Giant Planets. ApJ692:L9-L13

Lillo-Box J, Barrado D, Moya A et al. (2014) Kepler-91b: a planet at the end of its life. Planet and giant host star properties via light-curve variations. A\&A562:A109

Lin DNC Papaloizou J (1979) Tidal torques on accretion discs in binary systems with extreme mass ratios. MNRAS186:799-812

Lin DNC, Bodenheimer P Richardson DC (1996) Orbital migration of the planetary companion of 51 Pegasi to its present location. Nature380:606-607

Liu SF, Guillochon J, Lin DNC Ramirez-Ruiz E (2013) On the Survivability and Metamorphism of Tidally Disrupted Giant Planets: The Role of Dense Cores. ApJ762:37

Livio M Soker N (1984) Star-planet systems as possible progenitors of cataclysmic binaries. Monthly Notices of the Royal Astronomical Society (ISSN 0035-8711) 208:763-781

Mandell AM, Raymond SN Sigurdsson S (2007) Formation of Earth-like Planets During and After Giant Planet Migration. ApJ660:823-844

Mardling RA Lin DNC (2002) Calculating the Tidal, Spin, and Dynamical Evolution of Extrasolar Planetary Systems. ApJ573:829-844

Matsumura S, Takeda G Rasio FA (2008) On the Origins of Eccentric Close-In Planets. ApJ686:L29

McQuillan A, Mazeh T Aigrain S (2013) Stellar Rotation Periods of the Kepler Objects of Interest: A Dearth of Close-in Planets around Fast Rotators. The Astrophysical Journal Letters 775(1):L11

Meléndez J, Asplund M, Gustafsson B Yong D (2009) THE PECULIAR SOLAR COMPOSITION AND ITS POSSIBLE RELATION TO PLANET FORMATION. The Astrophysical Journal 704(1):L66-L70

Metzger BD, Giannios D Spiegel DS (2012) Optical and X-ray transients from planet-star mergers. MNRAS425:2778-2798

Murray CD Dermott SF (1999) Solar system dynamics

Mustill AJ, Davies MB Johansen A (2015) The Destruction of Inner Planetary Systems during High-eccentricity Migration of Gas Giants. ApJ808:14

Nagasawa M, Ida S Bessho T (2008) Formation of Hot Planets by a Combination of Planet Scattering, Tidal Circularization, and the Kozai Mechanism. ApJ678:498-508

Naoz S (2016) The Eccentric Kozai-Lidov Effect and Its Applications. ARA\&A54:441-489

Nordhaus J Blackman EG (2006) Low-mass binary-induced outflows from asymptotic giant branch stars. MNRAS370:2004-2012

Ogilvie GI (2014) Tidal Dissipation in Stars and Giant Planets. ARA\&A52:171-210

Paczyński B (1971) Evolutionary Processes in Close Binary Systems. ARA\&A9:183

Paxton B, Bildsten L, Dotter A et al. (2011) Modules for Experiments in Stellar Astrophysics (MESA).

apjs 192:3, URL http://dx.doi.org/10.1088/0067-0049/192/1/3

Paxton B, Cantiello M, Arras P et al. (2013) Modules for Experiments in Stellar Astrophysics (MESA): Planets, Oscillations, Rotation, and Massive Stars. apjs 208:4, URL http://dx.doi.org/10.1088/0067-0049/208/1/4

Paxton B, Marchant P, Schwab J et al. (2015) Modules for Experiments in Stellar Astrophysics (MESA): Binaries, Pulsations, and Explosions. ApJS220:15 
Penev K, Jackson B, Spada F Thom N (2012) Constraining Tidal Dissipation in Stars from the Destruction Rates of Exoplanets. ApJ751:96

Priedhorsky WC Verbunt F (1988) Tidal forces and mass transfer instabilities in low-mass x-ray binaries. Astrophys J 333:895-905, URL http://dx. doi .org/10.1086/166798

Ramírez I, Meléndez J Asplund M (2009) Accurate abundance patterns of solar twins and analogs. Does the anomalous solar chemical composition come from planet formation? A\&A 508(1):L17-L20

Rappaport S, Sanchis-Ojeda R, Rogers LA, Levine A Winn JN (2013) The Roche Limit for Closeorbiting Planets: Minimum Density, Composition Constraints, and Application to the $4.2 \mathrm{hr}$ Planet KOI 1843.03. ApJ773:L15

Rasio FA Ford EB (1996) Dynamical instabilities and the formation of extrasolar planetary systems. Science 274:954-956

Richardson DC, Bottke WF Love SG (1998) Tidal Distortion and Disruption of Earth-Crossing Asteroids. Icarus 134:47-76

Ritter H (1988) Turning on and off mass transfer in cataclysmic binaries. Astronomy and Astrophysics 202:93-100

Sackmann IJ Boothroyd AI (1999) Creation of ${ }^{7} \mathrm{Li}$ and Destruction of ${ }^{3} \mathrm{He},{ }^{9} \mathrm{Be},{ }^{1} \mathrm{OB}$, and ${ }^{1} 1 \mathrm{~B}$ in Low-Mass Red Giants, Due to Deep Circulation. The Astrophysical Journal 510(1):217-231

Sanchis-Ojeda R, Rappaport S, Winn JN et al. (2013) Transits and Occultations of an Earth-sized Planet in an 8.5 hr Orbit. ApJ774:54

Sanchis-Ojeda R, Rappaport S, Winn JN et al. (2014) A Study of the Shortest-period Planets Found with Kepler. ApJ787:47

Schlaufman KC Winn JN (2016) The Occurrence of Additional Giant Planets Inside the Water-Ice Line in Systems with Hot Jupiters: Evidence Against High-Eccentricity Migration. ApJ825:62

Setiawan J, Klement RJ, Henning T et al. (2010) A Giant Planet Around a Metal-Poor Star of Extragalactic Origin. Science 330:1642

Showman AP Guillot T (2002) Atmospheric circulation and tides of "51 Pegasus b-like" planets. A\&A385:166-180

Siess L Livio M (1999a) The accretion of brown dwarfs and planets by giant stars - I. Asymptotic giant branch stars. MNRAS 304(4):925-937

Siess L Livio M (1999b) The accretion of brown dwarfs and planets by giant stars - II. Solar-mass stars on the red giant branch. MNRAS 308(4):1133-1149

Skumanich A (1972) Time Scales for CA II Emission Decay, Rotational Braking, and Lithium Depletion. ApJ171:565

Stauffer JR Hartmann LW (1987) The distribution of rotational velocities for low-mass stars in the Pleiades. ApJ318:337-355

Steffen JH, Ragozzine D, Fabrycky DC et al. (2012) Kepler constraints on planets near hot Jupiters. Proceedings of the National Academy of Science 109:7982-7987

Teitler S Königl A (2014) Why is there a Dearth of Close-in Planets around Fast-rotating Stars? The Astrophysical Journal 786(2):139

Théado S Vauclair S (2012) Metal-rich Accretion and Thermohaline Instabilities in Exoplanet-host Stars: Consequences on the Light Elements Abundances. ApJ744:123

Trilling DE, Benz W, Guillot T et al. (1998) Orbital Evolution and Migration of Giant Planets: Modeling Extrasolar Planets. ApJ500:428-439

Tucci Maia M, Meléndez J Ramírez I (2014) High Precision Abundances in the 16 Cyg Binary System: A Signature of the Rocky Core in the Giant Planet. ApJ790:L25

Valsecchi F Rasio FA (2014) Planets on the Edge. ApJ787:L9

Valsecchi F, Rappaport S, Rasio FA, Marchant P Rogers LA (2015) Tidally-driven Roche-lobe Overflow of Hot Jupiters with MESA. ApJ813:101

van Eyken JC, Ciardi DR, von Braun K et al. (2012) The PTF Orion Project: A Possible Planet Transiting a T-Tauri Star. ApJ755:42

Van Laerhoven C Greenberg R (2012) Characterizing multi-planet systems with classical secular theory. Celestial Mechanics and Dynamical Astronomy 113:215-234 
Veras D, Leinhardt ZM, Bonsor A Gänsicke BT (2014) Formation of planetary debris discs around white dwarfs - I. Tidal disruption of an extremely eccentric asteroid. MNRAS445:2244-2255

Villaver E Livio M (2007) Can Planets Survive Stellar Evolution? ApJ661:1192-1201

Villaver E Livio M (2009) The Orbital Evolution of Gas Giant Planets Around Giant Stars. ApJ705:L81-L85

Wallerstein G Sneden C (1982) A K giant with an unusually high abundance of lithium - HD 112127. ApJ 255:577-584

Weiss LM Marcy GW (2014) The Mass-Radius Relation for 65 Exoplanets Smaller than 4 Earth Radii. ApJ783:L6

Winn JN, Fabrycky D, Albrecht S Johnson JA (2010) Hot Stars with Hot Jupiters Have High Obliquities. ApJ718:L145-L149

Zuckerman B, Koester D, Melis C, Hansen BM Jura M (2007) The Chemical Composition of an Extrasolar Minor Planet. The Astrophysical Journal 671(1):872-877 